\title{
Forma e tamanho dos frutos de pinhão-manso durante a secagem em cinco condições de ar
}

\author{
Valdiney C. Siqueira ${ }^{1}$, Osvaldo Resende ${ }^{2}$, Tarcísio H. Chaves $^{2}$ \& Frederico A. L. Soares ${ }^{2}$
}

\section{RESUMO}

Com base na necessidade de combustíveis que substituam o petróleo, do destaque do pinhão-manso na produção de biodiesel e da necessidade de construção e aperfeiçoamento dos equipamentos utilizados nas fases de colheita e pós-colheita desta cultura objetivou-se, com o presente trabalho, determinar a forma e o tamanho dos frutos de pinhão-manso durante a secagem em cinco condições de ar. Os frutos colhidos manualmente com o teor de água de 4,40 ( $\mathrm{kg}$ de água por $\mathrm{kg}$ de matéria seca) foram submetidos a secagem em estufa com circulação de ar forçada em cinco condições de temperatura, 45, 60, 75, 90 e $105{ }^{\circ} \mathrm{C}$, e umidades relativas de 14,$5 ; 7,4 ; 3,8 ; 2,2$ e $1,4 \%$, respectivamente, até atingir teor de água de $0,10 \pm 0,007$ ( $\mathrm{kg} \mathrm{kg}^{-1}$ de matéria seca). Foram determinados circularidade, esfericidade, volume, área superficial, área projetada e relação superfície-volume, ao longo da secagem. Conclui-se que a redução do teor de água e as condições de secagem alteram a forma e reduzem o tamanho dos frutos de pinhãomanso sendo possível ajustar uma única equação aos dados experimentais do volume e da área superficial dos frutos de pinhão-manso ao longo da secagem, em diversas temperaturas.

Palavras-chave: biodiesel, pós-colheita, Jatropha curcas L., teor de água

\section{Shape and size of Jatropha fruit during drying under five ambient conditions}

\begin{abstract}
Due to the need for substitution of fuel oil, the importance of Jatropha for biodiesel and the need for construction and improvements of the equipment used during harvest and postharvest of this crop, this research aimed to determine the shape and the size of Jatropha fruit during drying under five ambient conditions. The fruits harvested manually with moisture content of $4.40 \mathrm{~kg} \mathrm{~kg}^{-1} \mathrm{dry}$ matter were dried in an oven with forced air ventilation in five temperature conditions: $45,60,75,90$ and $105{ }^{\circ} \mathrm{C}$ and relative humidities of $14.5,7.4,3.8,2.2$ and $1.4 \%$ respectively, until moisture content of $0.10 \pm 0.007\left(\mathrm{~kg} \mathrm{~kg}^{-1}\right.$ dry matter). The roundness, sphericity, volume, surface area, projected area and surface-volume ratio were determined during drying. It is concluded that the reduction of the moisture content and the drying conditions alter the shape and reduce the size of the fruit of Jatropha, being possible to adjust a single equation to the experimental data of volume and surface area of the fruit of Jatropha during drying at different temperatures.
\end{abstract}

Key words: biodiesel, postharvest, Jatropha curcas L., moisture content 


\section{INTRODUÇÃO}

O pinhão-manso (Jatropha curcas L.), atualmente em processo de domesticação, é uma espécie cultivada na América desde a época pré-colombiana e só nos últimos trinta anos começou a ser mais pesquisada agronomicamente (Saturnino et al., 2005).

O óleo presente nas sementes de pinhão-manso tem sido amplamente estudado como alternativa para a produção de biocombustível. Souza et al. (2009) observaram uma concentração média de 40,33 $\pm 1,91 \%$ de lipídios na matéria seca de sementes de pinhão-manso. Outros atributos estão relacionados ao óleo que não é comestível não concorrendo, portanto, com a alimentação humana (Saturnino et al., 2005).

Santos et al. (2009) destacam que a distribuição geográfica do pinhão-manso é vasta pela sua rusticidade, resistência a longas estiagens, a pragas e doenças, sendo adaptável a condições edafoclimáticas muito variáveis, além da produção de óleo com todas as qualidades necessárias para ser transformado em óleo diesel.

A maior parte do cultivo do pinhão-manso ainda é realizada em pequenas lavouras em que os processos de colheita, secagem e armazenagem são feitos com recursos tecnológicos reduzidos. No entanto, com o advento do biodiesel novas tecnologias deverão ser adotadas para atender às necessidades de uma produção em larga escala (Santos et al., 2009). Sendo assim, para o desenvolvimento dos maquinários utilizados nas etapas de colheita e pós-colheita são de fundamental importância a determinação e o conhecimento do comportamento das propriedades físicas dos frutos de pinhãomanso.

Os materiais biológicos têm características físicas empregadas no desenvolvimento de projetos de máquinas e equipamentos; entretanto, torna-se necessário considerar que um material biológico não tem a uniformidade dos produtos confeccionados e moldados pela indústria química, mecânica e/ou elétrica (Cavalcanti-Mata \& Duarte, 2002).

As propriedades físicas do fruto do pinhão-manso são essenciais para os projetos de equipamentos para colheita, secagem, limpeza, classificação, descasque e armazenamento (Pradhan et al., 2009). Segundo Silva (2008), o conhecimento das propriedades físicas dos produtos agrícolas também é de extrema relevância para a adaptação de equipamentos já existentes visando obter maior rendimento nas operações de processamento.

De modo geral, os grãos e frutos apresentam formato geométrico indefinido tornando necessário, para a solução de problemas relacionados à sua geometria assumir, para o produto, uma forma conhecida, o que acarreta aproximações e possíveis erros (Corrêa et al., 2002).

Segundo Sirisomboon et al. (2007), o tamanho (área superficial, área projetada e volume) e a forma (circularidade, esfericidade) dos frutos, nozes e sementes, são imprescindíveis para seu processo de descasque. Esses dados podem ser usados para determinar o limite inferior do tamanho dos transportadores, como esteira, elevador de canecas e transportador helicoidal. A porosidade, área superficial específica e superfície, afetam a resistência à passagem do fluxo de ar através da camada de material, sendo relevante a geração de informações sobre tais aspectos para a melhor descrição do processo de secagem.

Segundo Resende et al. (2005), além de provocar seu encolhimento, a redução do teor de água dos grãos influencia diretamente nas suas propriedades físicas durante o processo de secagem e, sendo a secagem uma das etapas mais importantes durante a fase pós-colheita, é imprescindível o conhecimento do comportamento das propriedades físicas dos frutos de pinhão-manso uma vez que a extração do óleo das sementes normalmente é realizada após a secagem.

Considerando a importância do processo de secagem e o reflexo dessa etapa pós-colheita nas propriedades físicas dos produtos agrícolas objetivou-se, com o presente trabalho, avaliar a forma e determinar o tamanho dos frutos de pinhãomanso por meio da circularidade, esfericidade, volume, área superficial, área projetada e relação superfície-volume ao longo da secagem, em diversas condições de ar, e verificar a possibilidade de utilização de um único modelo visando à descrição dos resultados.

\section{Material e MÉTOdos}

O experimento foi desenvolvido no Laboratório de Póscolheita de Produtos Vegetais do Instituto Federal de Educação, Ciência e Tecnologia Goiano - Campus Rio Verde (IF GoianoCampus Rio Verde). Foram utilizados frutos de pinhão-manso, com teor de água médio inicial de 4,40 (kg de água por kg de matéria seca), submetidos a secagem em estufa com ventilação de ar forçada em cinco condições de temperatura, 45, 60, 75, 90 e $105^{\circ} \mathrm{C}$, e umidades relativas de 14,$5 ; 7,4 ; 3,8 ; 2,2$ e $1,4 \%$, respectivamente, até atingirem o teor de água (Ta) de 0,10 \pm 0,007 ( $\mathrm{kg}$ de água por $\mathrm{kg}$ de matéria seca).

A redução do teor de água ao longo da secagem foi acompanhada do método gravimétrico (perda de massa), por meio de uma balança analítica com resolução de $0,01 \mathrm{~g}$, conhecendo-se o teor de água inicial do produto, até atingir o teor de água desejado.

O teor de água dos frutos foi determinado em estufa com ventilação de ar forçada mantida a $105 \pm 1{ }^{\circ} \mathrm{C}$ até massa constante, em três repetições (Brasil, 2009).

Foram utilizados 15 frutos de pinhão-manso para cada condição de secagem, sendo os frutos individualizados em cápsulas de alumínio, com dimensões de $60,12 \times 10^{-3} \mathrm{~m}$ de diâmetro e $41,0 \times 10^{-3} \mathrm{~m}$ de altura. A determinação do comprimento, largura e espessura dos frutos foi realizada com o auxílio de um paquímetro digital com resolução de $0,01 \times 10^{-3}$ $\mathrm{m}$, quando eles se encontravam com os teores de água de 4,40; 2,$80 ; 1,98 ; 1,43 ; 1,05 ; 0,79 ; 0,58 ; 0,41 ; 0,28 ; 0,17$ e 0,10 , aproximadamente. A redução do teor de água foi acompanhada da pesagem de três repetições de $175 \mathrm{~g}$ de frutos de pinhãomanso mantidos nas mesmas condições de secagem, às quais os 15 frutos foram submetidos.

A forma dos frutos de pinhão-manso, considerados esferoides, foi obtida por meio da esfericidade e circularidade de acordo com as dimensões características expressas pelos eixos ortogonais (Figura 1). 


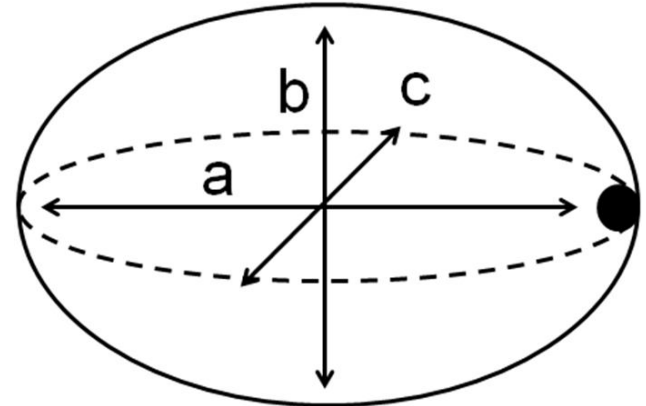

Obs.: a - maior eixo do fruto; b - eixo médio do fruto; c - menor eixo do fruto, todos em $\mathrm{m} 10^{-3}$ Figura 1. Desenho esquemático do fruto de pinhãomanso, considerado esferoide, com suas dimensões características

O diâmetro geométrico médio dos frutos de pinhão-manso foi calculado segundo a expressão proposta por Mohsenin (1986):

$$
\mathrm{D}_{\mathrm{g}}=(\mathrm{a} \times \mathrm{b} \times \mathrm{c})^{1 / 3}
$$

em que:

$\mathrm{D}_{\mathrm{g}}$ - diâmetro geométrico médio, $\mathrm{m} 10^{-3}$

A esfericidade $\left(\mathrm{E}_{\mathrm{s}}\right)$ dos frutos de pinhão-manso, dada em porcentagem, foi determinada de acordo com a seguinte expressão (Mohsenin, 1986):

$$
E_{S}=\left(\frac{D_{g}}{a}\right) \times 100
$$

A circularidade $(C)$ dos frutos de pinhão-manso na posição natural de repouso foi obtida utilizando a expressão a seguir, proposta por Mohsenin (1986):

$$
\mathrm{C}=\frac{\mathrm{b}}{\mathrm{a}} \times 100
$$

A área superficial $(\mathrm{S})$ em $\mathrm{m} 10^{-6}$ foi calculada pela analogia a uma esfera de mesmo diâmetro geométrico médio, usando a seguinte expressão (Tunde-Akinntunde \& Akintunde, 2004):

$$
S=\pi \times D_{g}
$$

A área projetada $\left(\mathrm{A}_{\mathrm{p}}\right) \mathrm{em} \mathrm{m}^{2} 10^{-6}$ dos frutos de pinhão-manso foi determinada pela seguinte expressão (Goneli et al., 2008):

$$
A_{p}=\frac{\pi a b}{4}
$$

$\mathrm{O}$ volume de cada fruto $\left(\mathrm{V}_{\mathrm{g}}\right)$ em $\mathrm{em}^{3} 10^{-9}$ foi obtido ao longo do processo de secagem de acordo com a expressão proposta por Mohsenin (1986):

$$
\mathrm{V}_{\mathrm{g}}=\frac{\pi \mathrm{abc}}{6}
$$

A relação superfície-volume (SV) foi calculada por meio da expressão seguinte:

$$
\mathrm{SV}=\frac{\mathrm{S}}{\mathrm{V}_{\mathrm{g}}}
$$

O experimento foi montado em esquema fatorial $5 \times 8$ (5 condições de secagem e 8 teores de água), em delineamento inteiramente casualizado, com três repetições. Os dados foram analisados por meio de análise de variância e regressão e os modelos selecionados de acordo com o coeficiente de determinação e pela significância da equação pelo teste $\mathrm{F}$. Depois da seleção do modelo para a representação das propriedades físicas dos frutos de pinhão-manso, promoveuse o teste da hipótese de igualdade dos modelos utilizando-se a técnica de identidade de modelos, descrita por Regazzi (2003), que permite analisar a equivalência entre os modelos utilizados objetivando o ajuste de uma única equação para descrição do fenômeno estudado, por meio do teste de qui-quadrado $\left(\mathrm{c}^{2}\right)$.

Os coeficientes dos modelos linear $\left(a_{i}, b_{i}\right)$ e polinomial $\left(a_{i}, b_{i}\right.$ e $c_{i}$ ), utilizados para descrever a esfericidade, circularidade, volume, área superficial, área projetada e relação superfícievolume, foram comparados entre si para verificar sua igualdade. As seguintes hipóteses foram formuladas:

Modelo linear:

$$
\mathrm{H}_{\mathrm{o}}^{(1)}: \mathrm{a} 1=\mathrm{a} 2=\mathrm{a} 3=\mathrm{a} 4=\mathrm{a} 5 \text { e b1 = b2 = b3 = b4 = b5 }
$$

versus

$\mathrm{H}_{\mathrm{a}}^{(1)}$ - existe pelo menos uma desigualdade entre os parâmetros

Modelo polinomial:

$$
\mathrm{H}_{\mathrm{o}}^{(2)}-\mathrm{a} 1=\mathrm{a} 2=\mathrm{a} 3=\mathrm{a} 4=\mathrm{a} 5 ; \mathrm{b} 1=\mathrm{b} 2=\mathrm{b} 3=\mathrm{b} 4=\mathrm{b} 5
$$

e

$$
\mathrm{c} 1=\mathrm{c} 2=\mathrm{c} 3=\mathrm{c} 4=\mathrm{c} 5
$$

versus

$\mathrm{H}_{\mathrm{a}}^{(2)}$ - existe pelo menos uma desigualdade entre os parâmetros.

\section{RESULTADOS E DISCUSSÃO}

Verifica-se, na Figura 2A, que no início do processo de secagem os frutos de pinhão-manso se encontram com formato semelhante ao de uma esfera e que, com a secagem, a esfericidade diminui para todas as condições de temperatura. Este comportamento vem sendo observado por diversos pesquisadores para vários produtos (Sacilik et al., 2003; Yalçin \& Ozarshan, 2004; Karababa, 2006). Com a redução do teor de água de 4,40 para 0,10 ( $\mathrm{kg}$ de água por kg de matéria seca), houve redução na esfericidade de 9,$17 ; 8,21 ; 6,34 ; 7,03$ e 7,11 pontos percentuais para as temperaturas de 45, 60, 75, 90 e 105 ${ }^{\circ} \mathrm{C}$, respectivamente, evidenciando que a esfericidade dos 
A.

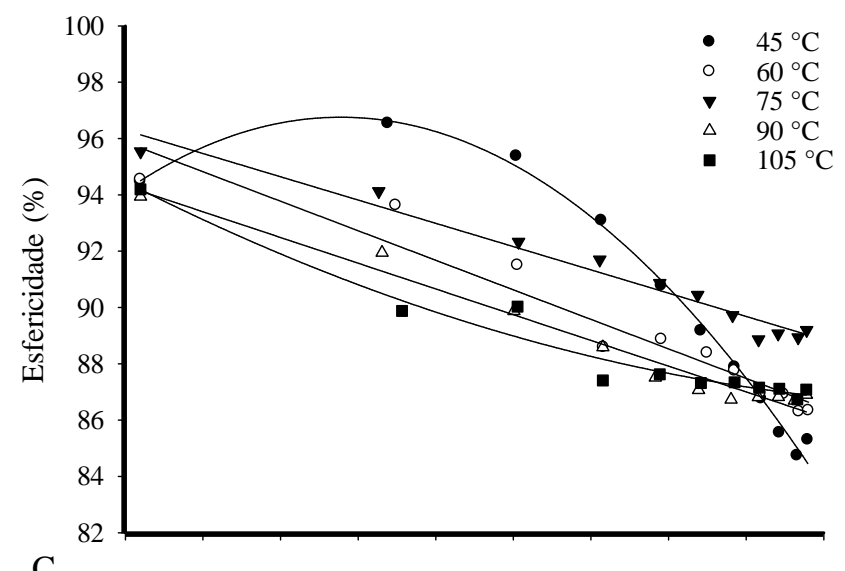

C.

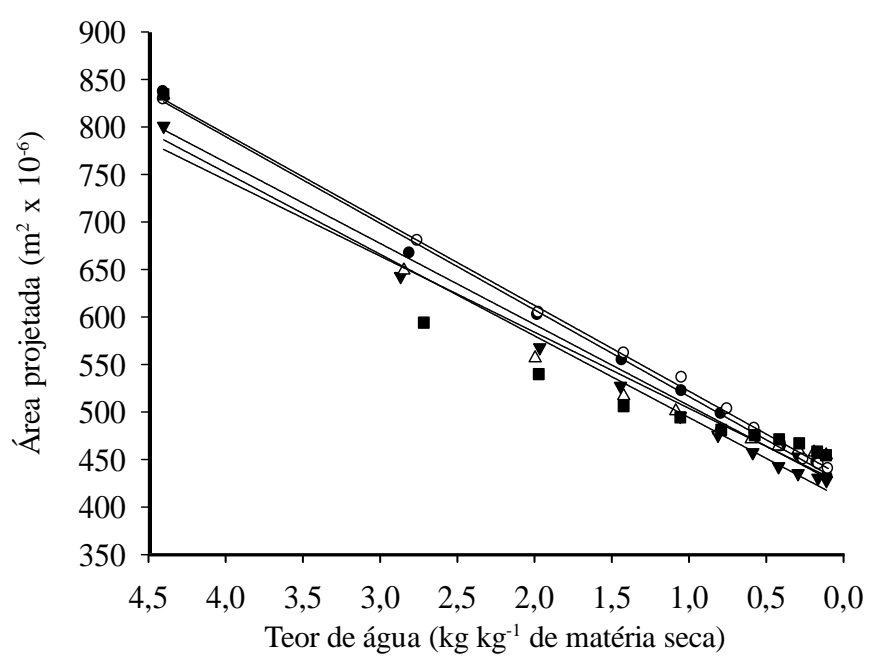

B.

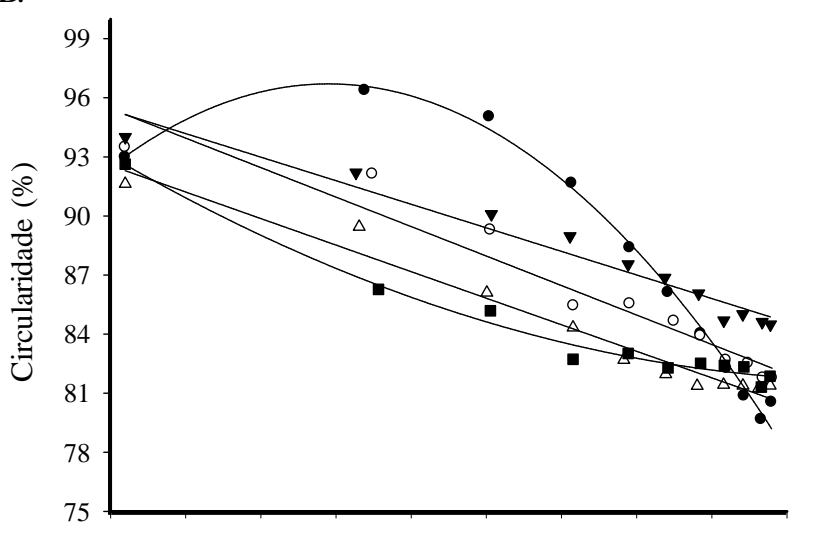

D.

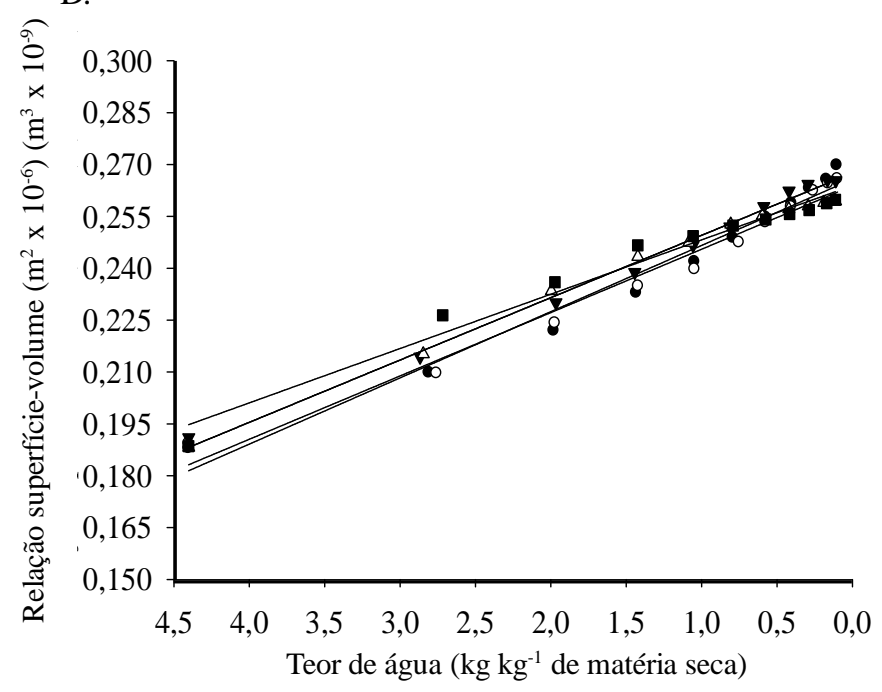

Figura 2. Esfericidade (A), circularidade (B), área projetada (C) e relação superfície-volume (D) dos frutos de pinhãomanso submetidos à secagem em cinco condições de ar

frutos de pinhão-manso é mais afetada quando eles são submetidos à secagem com temperaturas mais baixas, comportamento que pode estar relacionado à baixa taxa de remoção de água nessas condições de secagem.

Ainda na Figura 2A, nota-se que no início do processo de secagem a esfericidade dos frutos de pinhão-manso se encontra com um valor médio de $94,5 \%$ e, ao final, de $86,9 \%$. Resultados semelhantes foram relatados por Sirisomboon et al. (2007), uma vez que os frutos de pinhão-manso com o teor de água de 8,05 ( $\mathrm{kg}$ de água por $\mathrm{kg}$ de matéria seca) apresentaram uma esfericidade de $95 \pm 3 \%$, e nos resultados obtidos por Pradan et al. (2009), a esfericidade dos frutos de pinhão-manso apresentou o valor de $83 \pm 3,4 \%$ para a faixa de teor de água de 0,08 a 0,30 ( $k g$ de água por $\mathrm{kg}$ de matéria seca), respectivamente.

Nota-se, na Figura 2B, que a circularidade dos frutos de pinhão-manso apresenta comportamento semelhante ao da esfericidade (Figura 2A), ou seja, diminui com a redução do teor de água. Resultados semelhantes foram observados por Corrêa et al. (2002), trabalhando com frutos de café Coffea arabica e C. canephora.

Goneli et al. (2011), analisando a circularidade e a esfericidade dos frutos de mamona, planta pertencente à mesma família do pinhão-manso, verificaram que com a redução do teor de água de 2,492 para 0,132 ( $\mathrm{kg}$ de água por kg de matéria seca), a circularidade e a esfericidade foram reduzidas de 96,17 para $91,84 \%$ e de 95,02 para $92,77 \%$, respectivamente.

Pode-se observar na Figura 2B que, proporcionalmente, há maior redução no eixo médio em relação ao maior eixo. No entanto, assim como na esfericidade a circularidade dos frutos de pinhão-manso na temperatura de $45^{\circ} \mathrm{C}$ aumenta, segundo o modelo ajustado, até um teor de 3,11 e 3,05 kg de água por $\mathrm{kg}$ de matéria seca, para esfericidade e circularidade, respectivamente, diminuindo a partir daí, indicando que quando frutos muito úmidos são submetidos a uma baixa taxa de remoção de água, a proporção da contração da largura em relação ao comprimento é menor.

Segundo Mohsenin (1986), a circularidade indica a agudeza dos cantos de um sólido; sendo assim, a projeção dos frutos de pinhão-manso pode ser satisfatoriamente representada por um círculo, sobretudo quando os frutos se encontram com alto teor de água.

Observa-se na Figura 2C que a área projetada dos frutos de pinhão-manso decresce com a redução do teor de água apresentando, no final do processo de secagem, uma diferença de $379,75 \times 10^{-6} ; 378,89 \times 10^{-6} ; 372,74 \times 10^{-6} ; 388,65 \times 10^{-6} \mathrm{e} 404,91$ $\mathrm{x} 10^{-6} \mathrm{~m}^{2} \mathrm{em}$ relação à área projetada inicial, para as temperaturas 
de $105,90,75,60$ e $45^{\circ} \mathrm{C}$, respectivamente. A redução da área projetada em função da diminuição do teor de água também vem sendo observada por diversos pesquisadores para vários produtos (Aydin, 2007; Dursun et al., 2007; Yalçin et al., 2007).

Verifica-se na Figura 2D que, para as cinco condições de secagem analisadas, a redução do teor de água aumenta a relação superfície-volume. Segundo Farinha (2008), se apenas fatores físicos estiverem envolvidos, a taxa de redução de água é proporcional à relação superfície-volume e admitindo que a forma do produto seja constante, a relação superfície-volume aumenta com a redução do tamanho.

Observa-se na Figura 3A que na redução do teor de água de 4,40 para $0,10 \pm 0,007\left(\mathrm{~kg} \mathrm{~kg}^{-1}\right.$ de matéria seca), ocorreu acentuada diminuição do volume dos frutos, com decréscimo de 10512,$1 ; 10602,97 ; 10235,32 ; 10772,04$ e 11352,27 m 10 $10^{-9} \mathrm{em}$ relação ao volume inicial, para as temperaturas de 105, 90, 75, 60 e $45^{\circ} \mathrm{C}$, respectivamente.

Nota-se ainda, na Figura $3 \mathrm{~A}$ que, para as temperaturas mais altas $\left(105,90\right.$ e $\left.75^{\circ} \mathrm{C}\right)$, a redução do volume foi menor possivelmente pela elevada taxa de remoção de água, ocasionando enrijecimento do tegumento, que dificultou a contração dos frutos e, em consequência, reduziu com menor intensidade seu volume. Nas temperaturas mais baixas (60 e 45 ${ }^{\circ} \mathrm{C}$ ), a redução do volume inicial foi maior uma vez que a água foi removida mais lentamente e os frutos contraíram os eixos ortogonais de acordo com a redução do teor de água.

Verifica-se na Figura 3B que a área superficial dos frutos de pinhão-manso diminuiu com a secagem estando seu

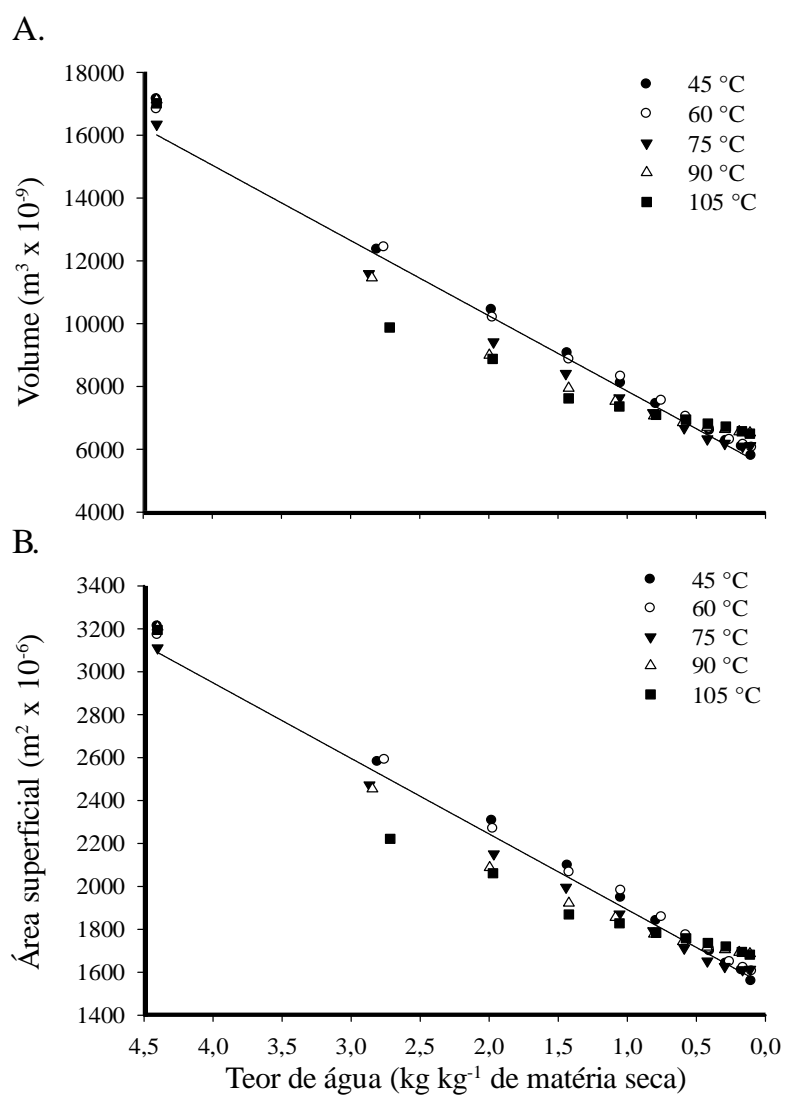

Figura 3. Volume (A) e área superficial (B) dos frutos de pinhão-manso submetidos a diversas condições de secagem comportamento intimamente relacionado ao do volume, sendo bastante influenciado pelo teor de água do fruto. Assim como na Figura 3A, nota-se que as condições do ar de secagem praticamente não influenciam a área superficial do produto, sendo este comportamento restrito ao teor de água, uma vez que foi possível a utilização de uma única equação para representar todas as cinco condições de ar utilizadas. Karababa (2006) verificou um decréscimo de 40,16\% da área superficial dos grãos de milho pipoca com a redução do teor de água de 0,20 para 0,098 ( $\mathrm{kg}$ de água por kg de matéria seca). Goneli et al. (2008) observaram decréscimo de $496 \mathrm{~m} .10^{-6}$, aproximadamente, com a redução do teor de água de 2,5 para 0,13 (kg de água por $\mathrm{kg}$ de matéria seca), em frutos de mamona.

Ainda na Figura 3B observa-se que o valor médio inicial de área superficial dos frutos de pinhão-manso foi de 3161,39 \pm $50,7 \mathrm{~m}^{2} 10^{-6}$. Esses resultados são semelhantes aos valores reportados por Sirisomboon et al. (2007) em que os frutos de pinhão-manso com o teor de água de 8,05 ( $\mathrm{kg}$ de água por $\mathrm{kg}$ de matéria seca) apresentaram uma área superficial de 3139,21 $\pm 197,41 \mathrm{~m}^{2} 10^{-6}$. O valor médio final de área superficial dos frutos de pinhão-manso foi de $1622,70 \pm 63,69 \mathrm{~m} \mathrm{10}^{-6}$, inferior aos resultados apresentados por Pradan et al. (2009), em que a área superficial dos frutos de pinhão-manso variou de 1815,73 a $1917,59 \mathrm{~m}^{2} 10^{-6}$ para a faixa de teor de água de 0,08 a $0,30(\mathrm{~kg}$ de água por kg de matéria seca), respectivamente.

Na Tabela 1 estão apresentados os resultados do teste das hipóteses analisadas pelo teste de qui-quadrado para as combinações dos parâmetros estimados pelo modelo Linear para volume, área superficial, área projetada e relação superfícievolume e o modelo Polinomial para esfericidade e circularidade dos frutos de pinhão-manso submetidos a secagem em diferentes temperaturas.

Tabela 1. Hipóteses avaliadas $\mathrm{H}_{\mathrm{o}}$ e $\mathrm{H}_{\mathrm{a}^{\prime}}$ valores da estatística do teste qui-quadrado $\left(\chi^{2}\right)$ a 0,05 de probabilidade e número de graus de liberdade $(v)$ para esfericidade (\%), circularidade $(\%)$, volume $\left(\mathrm{m}^{3} \times 10^{-9}\right)$, área superficial $\left(\mathrm{m}^{2}\right.$ $\left.\times 10^{-6}\right)$, área projetada $\left(\mathrm{m}^{2} \times 10^{-6}\right)$ e relação superfícievolume $\left(\mathrm{m}^{2} \times 10^{-6} / \mathrm{m}^{3} \times 10^{-9}\right)$ dos frutos de pinhão-manso ao longo da secagem em cinco condições de ar

\begin{tabular}{|c|c|c|c|c|}
\hline Variável & Hipótese & GL $(v)$ & $\chi_{(0,05 ; v)}^{2}$ & $\chi_{\text {calculado }}^{2}$ \\
\hline Esfericidade & $\mathrm{H}_{0}^{\left({ }^{(2)}\right.}$ versus $\mathrm{H}_{\mathrm{a}}{ }^{(2)}$ & 12 & 21,03 & $-109,68$ * \\
\hline Circularidade & $\mathrm{H}_{0}^{(2)}$ versus $\mathrm{H}_{\mathrm{a}}^{(2)}$ & 12 & 21,03 & 27,97 * \\
\hline Volume & $\mathrm{H}_{0}^{(1)}$ versus $\mathrm{H}_{\mathrm{a}}^{(1)}$ & 8 & 15,50 & $10,51^{\mathrm{ns}}$ \\
\hline Área superficial & $\mathrm{H}_{0}^{(1)}$ versus $\mathrm{H}_{\mathrm{a}}^{(1)}$ & 8 & 15,50 & $15,31^{\mathrm{ns}}$ \\
\hline Área projetada & $\mathrm{H}_{0}^{(1)}$ versus $\mathrm{H}_{\mathrm{a}}^{(1)}$ & 8 & 15,50 & 20,54 * \\
\hline Relação superfície-volume & $\mathrm{H}_{0}^{(1)}$ versus $\mathrm{H}_{\mathrm{a}}^{(1)}$ & 8 & 15,50 & 21,36 * \\
\hline
\end{tabular}

Verifica-se, na Tabela, 1 que os valores de $\chi_{\text {calculados }}^{2}$ foram superiores aos valores de $\chi_{\text {tabelados }}^{2}$ para área projetada, relação superfície-volume, esfericidade e circularidade. Assim, rejeitamse as hipóteses formuladas $\mathrm{H}_{\mathrm{o}}^{(1)} \mathrm{e}_{\mathrm{o}}^{(2)}$, ou seja, os modelos analisados para a representação dessas propriedades físicas dos frutos de pinhão-manso diferem estatisticamente entre si. Entretanto, analisando o volume e a área superficial, observase que o valor de $\chi_{\text {calculado }}^{2}$ foi inferior ao valor de $\chi_{\text {tabelado' }}^{2}$, 
Tabela 2. Equações ajustadas aos valores de esfericidade, circularidade, área projetada e relação superfície-volume dos frutos de pinhão-manso em função do teor de água

\begin{tabular}{|c|c|c|c|c|}
\hline \multirow{2}{*}{$\mathrm{T}\left({ }^{\circ} \mathrm{C}\right)$} & \multicolumn{2}{|l|}{ Esfericidade } & \multicolumn{2}{|l|}{ Circularidade } \\
\hline & Modelo & $\mathbf{R}^{2}(\%)$ & Modelo & $\mathbf{R}^{2}(\%)$ \\
\hline $\begin{array}{r}105 \\
90 \\
75 \\
60 \\
45\end{array}$ & $\begin{array}{l}E_{s}=86,82+0,591 \mathrm{Ta}+0,245 \mathrm{Ta}^{2} \\
E_{s}=86,08+1,828 \mathrm{Ta} \\
E_{s}=88,85+1,653 \mathrm{Ta} \\
E_{s}=86,43+2,097 \mathrm{Ta} \\
E_{s}=83,60+8,450 \mathrm{Ta}-1,357 \mathrm{Ta}^{2}\end{array}$ & $\begin{array}{l}96,0^{\star \star} \\
97,1^{\star \star} \\
96,7^{\star \star} \\
93,5^{\star \star} \\
99,3^{\star \star}\end{array}$ & $\begin{array}{l}\mathrm{C}=81,79+0,548 \mathrm{Ta}+0,433 \mathrm{Ta}^{2} \\
\mathrm{C}=80,43+2,696 \mathrm{Ta} \\
\mathrm{C}=84,61+2,393 \mathrm{Ta} \\
\mathrm{C}=81,97+2,993 \mathrm{Ta} \\
C=77,95+12,29 \mathrm{Ta}-2,015 \mathrm{Ta}^{2}\end{array}$ & $\begin{array}{l}99,2^{\star *} \\
93,8^{* *} \\
95,5^{* *} \\
96,8^{* *} \\
98,4^{* *}\end{array}$ \\
\hline \multirow{2}{*}{$\mathrm{T}\left({ }^{\circ} \mathrm{C}\right)$} & \multicolumn{2}{|l|}{$\begin{array}{l}\text { Área projetada } \\
\end{array}$} & \multicolumn{2}{|c|}{ Relação superfície-volume } \\
\hline & Modelo & $\mathbf{R}^{2}(\%)$ & Modelo & $R^{2}(\%)$ \\
\hline $\begin{array}{r}105 \\
90 \\
75 \\
60 \\
45\end{array}$ & $\begin{array}{l}A_{p}=423,8+80,08 \mathrm{Ta} \\
A_{p}=421,4+85,33 \mathrm{Ta} \\
A_{p}=408,3+85,91 \mathrm{Ta} \\
A_{p}=431,7+90,23 \mathrm{Ta} \\
A_{p}=425,29+91,12 \mathrm{Ta}\end{array}$ & $\begin{array}{l}91,7^{\star \star} \\
96,3^{\star \star} \\
99,5^{\star \star} \\
99,8^{\star \star} \\
99,7^{\star \star}\end{array}$ & $\begin{array}{l}S V=0,263-0,015 T a \\
S V=0,264-0,016 T a \\
S V=0,267-0,018 T a \\
S V=0,263-0,018 T a \\
S V=0,265-0,019 T a\end{array}$ & $\begin{array}{l}97,1^{* *} \\
99,1^{* *} \\
99,3^{* *} \\
97,6^{* *} \\
97,2^{* *}\end{array}$ \\
\hline
\end{tabular}

Significativo a 0,01 de probabilidade, pelo teste $\mathrm{F}$

aceitando-se a hipótese $\mathrm{H}_{\mathrm{o}}^{(1)}$, possibilitando a utilização de um único modelo para a descrição dessas variáveis.

O volume e a área superficial dos frutos de pinhão-manso durante a secagem em cinco condições de temperatura podem ser representados pelas Eqs. 8 e 9, respectivamente, de acordo com o teste de qui-quadrado a nível de $5 \%$ de probabilidade.

$$
\begin{gathered}
V_{g}=5488,670+2391,284 T_{a} \\
S=1544,268+351,3366 T_{a}
\end{gathered}
$$

Na Tabela 2 são apresentados os modelos ajustados aos dados experimentais de esfericidade, circularidade, área projetada e relação superfície-volume dos frutos de pinhão-manso em função do teor de água. Observa-se que os modelos apresentaram alto grau de significância de acordo com o teste $\mathrm{F}$ e valores do coeficiente de determinação superiores a 91\%. Assim, referidos modelos descrevem, de forma satisfatória, o comportamento das variáveis analisadas em função do teor de água.

O ajuste de modelos lineares e polinomiais a essas características físicas de produtos vegetais vem sendo observado por diversos pesquisadores (Baryeh, 2002; Baryeh \& Mangope, 2002; Nimkar et al., 2005; Karababa, 2006; Garnayak et al., 2008; Goneli et al., 2008; Pradhan et al., 2009).

\section{CONCLUSÕES}

1. A redução do teor de água e as condições de secagem alteram a forma e reduzem o tamanho dos frutos de pinhãomanso, sendo a influência da temperatura restrita à circularidade e à esfericidade.

2. O processo de secagem reduz a esfericidade, a circularidade, o volume, a área superficial, a área projetada e aumenta a relação superfície-volume dos frutos de pinhãomanso.

3. É possível ajustar significativamente uma única equação aos dados experimentais do volume e da área superficial dos frutos de pinhão-manso ao longo da secagem em diversas temperaturas.

\section{LITERATURA CITADA}

Aydin, C. Some engineering properties of peanut and kernel. Journal of Food Engineering, v.79, p.810-816, 2007.

Baryeh, E. A. Physical properties of millet. Journal of Food Engineering, v.51, p.39-46, 2002.

Baryeh, E. A.; Mangope, B. K. Some physical properties of QP38 variety pigeon pea. Journal of Food Engineering, v.56, p.59-65, 2002.

Brasil. Ministério da Agricultura Pecuária e Abastecimento. Secretaria de defesa agropecuária. Regras para análise de sementes. Brasília: MAPA/ACS, 2009. 395p.

Cavalcanti-Mata, M. E. R. M.; Duarte, M. E. M. Porosidade intergranular de produtos agrícolas. Revista Brasileira de Produtos Agroindustriais, v.4, p.79-93, 2002.

Corrêa, P. C.; Afonso Júnior, P. C.; Queiroz, D. M.; Sampaio, C. P.; Cardoso, J. B. Variação das dimensões características e da forma dos frutos de café durante o processo de secagem. Revista Brasileira de Engenharia Agrícola e Ambiental, v.6, p.466-470, 2002.

Dursun, I.; Tuðrul, K. M.; Dursun, E. Some physical properties of sugarbeet seed. Journal of Stored Products Research, v.43, p.149-155, 2007.

Farinha, L. R. L. Características físicas na perda da matéria fresca e evolução das propriedades reológicas e cor da cenoura cv. Brasília no armazenamento. Viçosa: UFV, 2008. 111p. Dissertação Mestrado

Garnayak, D. K.; Pradhan, R. C.; Naik, S. N.; Bhatnagar N. Moisture-dependent physical properties of Jatropha seed (Jatropha curcas L.), Industrial Crops and Products, v.27, p.123-129, 2008.

Goneli, A. L. D.; Corrêa, P. C.; Botelho, F. M.; Oliveira, G. H. H.; Santos, E. S. Propriedades físicas dos frutos de mamona durante a secagem. Revista Brasileira de Armazenamento, v.33, p.148-155, 2008.

Goneli, A. L. D.; Corrêa, P. C.; Magalhães, F. E. A.; Baptestini, F. M. Contração volumétrica e forma dos frutos de mamona durante a secagem. Acta Scientiarum. Agronomy, v.33, p.18, 2011.

Karababa, E. Physical properties of popcorn kernels. Journal of Food Engineering, v.72, p.100-107, 2006. 
Mohsenin, N. N. Physical properties of plant and animal materials. New York: Gordon and Breach Publishers, 1986. 841p.

Nimkar, P. M.; Mandwe, D. S.; Dudhe, R. M. Physical properties of Moth Gram. Biosystems Engineering, v.91, p.183-189, 2005.

Pradhan, R. C.; Naik, S. N.; Bhatnagar, N.; Vijay V. K. Moisturedependent physical properties of jatropha fruit. Industrial Crops and Products, v.29, p.341-347. 2009.

Regazzi, A. J. Teste para verificar a igualdade de parâmetros e a identidade de modelos de regressão não linear. Revista Ceres, v.50, p.9-26, 2003.

Resende, O.; Corrêa, P. C.; Goneli, A. L. D.; Cecon, P. R. Forma, tamanho e contração volumétrica do feijão (Phaseolus vulgaris L.) durante a secagem. Revista Brasileira de Produtos Agroindustriais, v.7, p. 15-24, 2005.

Sacilik, K.; Ozturk, R.; Keskin, R. Some physical properties of hemp seed. Biosystems Engineering, v.86, p.191-198, 2003.

Santos, S. B.; Martins, M. A.; Carvalho, F. M.; Carneiro, A. C. O. Determinação de algumas propriedades físicas dos grãos de pinhão manso (Jatropha curcas L.) In: Di Leo, N.; Montico, S.; Nardón, G. Avances en Ingeniería Rural: 2007 2009. Rosário: UNR, 2009. p.1067-1072.
Saturnino, H. M.; Kakida, D. D. P. J.; Tominaga, N.; Gonçalves, N. P. Cultura do pinhão-manso (Jatropha curcas L.). Informe Agropecuário, v.26, p.44-78, 2005.

Silva, J. S. Secagem e armazenagem de produtos agrícolas, Viçosa: Aprenda Fácil, 2008. 559p.

Sirisomboon, P.; Kitchaiya, P.; Pholpho, T.; Mahuttanyavanitch, W. Physical and mechanical properties of Jatropha curcas L. fruits, nuts and kernels. Biosystems Engineering, v.97, p.201-207, 2007.

Souza, A. D. V.; Fávaro, S. P.; Ítavo, L. C. V.; Roscoe, R. Caracterização química de sementes e tortas de pinhãomanso, nabo-forrageiro e crambe. Pesquisa Agropecuária Brasileira, v.44, p.1328-1335, 2009.

Tunde-Akinntunde, T. Y.; Akintunde, B. O. Some physical properties of sesame seed. Biosystems Engineering, v.88, p.127-129, 2004.

Yalçin, Y.; Özarslan, C. Physical properties of vetch seed. Biosystems Engineering, v.88, p.507-512, 2004.

Yalçin, Ý.; Özarslan, C.; Akbap, T. Physical properties of pea (Pisum sativum) seed. Journal of Food Engineering, v.79, p.731-735, 2007. 